\title{
Genetic susceptibility to bilateral tinnitus in a Swedish twin cohort
}

\author{
Iris Lianne Maas, MSc'1, Petra Brüggemann, $\mathrm{PhD}^{2}$, Teresa Requena, $\mathrm{PhD}^{3}$, Jan Bulla, $\mathrm{PhD}^{4}$, \\ Niklas K. Edvall, MSc ${ }^{5}$, Jacob v.B. Hjelmborg, PhD ${ }^{6}$, Agnieszka J. Szczepek, PhD ${ }^{7}$, Barbara Canlon, PhD \\ Birgit Mazurek, MD, PhD2, Jose A. Lopez-Escamez, MD, PhD ${ }^{3,8}$ and Christopher R. Cederroth, PhD ${ }^{5}$
}

Purpose: Genetic contributions to tinnitus have been difficult to determine due to the heterogeneity of the condition and its broad etiology. Here, we evaluated the genetic and nongenetic influences on self-reported tinnitus from the Swedish Twin Registry (STR).

Methods: Cross-sectional data from the STR was obtained. Casewise concordance rates (the risk of one twin being affected given that his/her twin partner has tinnitus) were compared for monozygotic (MZ) and dizygotic (DZ) twin pairs $(N=10,464$ concordant and discordant twin pairs) and heritability coefficients (the proportion of the total variance attributable to genetic factors) were calculated using biometrical model fitting procedures.

Results: Stratification of tinnitus cases into subtypes according to laterality (unilateral versus bilateral) revealed that heritability of bilateral tinnitus was 0.56 ; however, it was 0.27 for unilateral tinnitus. Heritability was greater in men (0.68) than in women (0.41). However, when female pairs younger than 40 years of age were selected, heritability of 0.62 was achieved with negligible effects of shared environment.

Conclusion: Unlike unilateral tinnitus, bilateral tinnitus is influenced by genetic factors and might constitute a genetic subtype. Overall, our study provides the initial evidence for a tinnitus phenotype with a genetic influence.

Genet Med advance online publication 23 March 2017

Key Words: concordance; gender; heritability; tinnitus; twins

\section{INTRODUCTION}

Tinnitus, the phantom sensation of sound, is a common condition that is chronic in more than $15 \%$ of the population. Severe tinnitus-induced distress, which occurs in $1-2 \%$ of the population, interferes with sleep and work productivity. ${ }^{1-3}$ Although a gender bias in tinnitus is still uncertain, the majority of studies report that men are more often affected than women. ${ }^{4}$ The incidence of tinnitus increases with age and correlates with hearing loss and other age-related disorders (e.g. hypertension and diabetes). ${ }^{5}$ Moreover, tinnitus is considered by most clinicians to have a broad etiology, ranging from noise trauma to ototoxicity, hearing loss, stress, or Ménière disease. ${ }^{6}$ The tinnitus percept can be pulsatile, nonpulsatile, constant, or intermittent; it can be tonal, sound like buzzing, whooshing, or ringing, and it can affect one or both ears. ${ }^{6}$

The possibility of genetic factors contributing to the development of tinnitus has not been well explored. ${ }^{4}$ For instance, there is no clear evidence of a dependency of tinnitus prevalence on ethnic background or geographic area. Kvestad et al. reported sibling-sibling correlations ranging from 0.06 to $0.14(N=$ 11,498 siblings), ${ }^{7}$ consistent with another European multicenter study ( $N=981$ siblings) that identified a sibling-sibling correlation of $0.16 .^{8}$ In one study, the likelihood of developing tinnitus for persons with a sibling with tinnitus was 1.7 -fold higher than for individuals without. ${ }^{8}$ However, it was postulated that the presence of a family member with tinnitus raises awareness, consequently increasing the apparent prevalence among other members of the family.

Comparing the concordance of a disease in monozygotic twins versus dizygotic twins yields important information about the relative contribution of genetic and environmental factors to the disease etiology. Studies involving twins offer a powerful tool for the research of familial influences (genetic and shared early environment). In addition, differences between identical

\footnotetext{
The first four authors share equal authorship.

${ }^{1}$ Department of Psychosomatic Medicine, Center for Internal Medicine and Dermatology, Charité-Universitätsmedizin Berlin, Berlin, Germany; ${ }^{2}$ Tinnitus Center,

Charité-Universitätsmedizin Berlin, Berlin, Germany; ${ }^{3}$ Otology \& Neurotology Group CTS495, Department of Genomic Medicine, GENYO - Center for Genomics and Oncological Research-Pfizer, University of Granada, Junta de Andalucía, PTS, Granada, Spain; ${ }^{4}$ Department of Mathematics, University of Bergen, Bergen, Norway; ${ }^{5}$ Experimental Audiology, Department of Physiology and Pharmacology, Karolinska Institutet, Stockholm, Sweden; ${ }^{6}$ Department of Epidemiology, Biostatistics and Biodemography, University of Southern Denmark, Odense, Denmark; ${ }^{7}$ Department of ORL, Head and Neck Surgery, Research Laboratory, Charité-Universitätsmedizin Berlin, Berlin, Germany; ${ }^{8}$ Department of Otolaryngology, Complejo Hospitalario Universidad de Granada (CHUGRA), ibs.granada, Granada, Spain. Correspondence: Christopher R. Cederroth (christopher.cederroth@ki.se) or Jose Antonio Lopez-Escamez (antonio.lopezescamez@genyo.es)
} 
(monozygotic) and fraternal (dizygotic) twins provide important information regarding both genetic and environmental effects. To the best of our knowledge, there are no published concordance studies that focus on tinnitus. Monozygotic twins (MZ) share all of their alleles, whereas dizygotic twins (DZ) share, on average, half of their alleles. As a consequence, if a greater concordance of tinnitus is found in $\mathrm{MZ}$ than in $\mathrm{DZ}$, this would imply that genetic factors are important in tinnitus etiology. Moreover, inclusion of opposite sex (OS-DZ) twins would be essential to determine whether different sets of genes or different shared environments are operating in the two sexes. The aim of this study was to evaluate the importance of genetic and environmental factors on tinnitus in the Swedish Twin Registry.

\section{MATERIALS AND METHODS}

\section{Participants}

Participants were twins from the Swedish Twin Registry (STR) born between 1900 and 1985 and who participated in the Screening Across the Lifespan Twin study (SALT) and the Study of Twin Adults: Genes and Environment (STAGE). ${ }^{9}$ SALT is a screening program of all twins born in 1958 or earlier based on computer-assisted telephone interviews. STAGE is a Web-based survey of twins born in Sweden between 1959 and 1985. ${ }^{9}$ The youngest age of twins who participated in the project was 19 years. The project was approved by the local ethics committee, Regionala etikprövningsnämnden, in Stockholm (2015/2129-31/1), and the data request was approved by the steering committee of the STR.

\section{Cohort characteristics}

In total, 70,186 twin individuals from SALT and STAGE were presented with the question, in Swedish, "Do you have buzzing in the ears?" The possible answers were (i) No, (ii) Yes-one ear, (iii) Yes-two ears, and (iv) Don't know/refuse to answer. The source population represented various social groups: students (2.4\%), unemployed individuals (2.4\%), individuals on maternity/paternity leave (1.2\%), individuals on sick leave or disability pension (7.6\%), workers in various professions and sectors (45.2\% full-time employed; $12.1 \%$ part-time employed), retirees $(17.9 \%)$, and other occupations (3.2\%); social group data were missing for $8 \%$ of the subjects. Of the respondents, $14.9 \%$ reported having tinnitus, with an overall gender bias-males were significantly more often affected than women $(17.8 \%$ in men and $12.5 \%$ in women; $\left.\chi^{2}(1)=365.57 ; P<0.001\right)$. In the younger age groups ( $\leq 24$ years), tinnitus prevalence was $14.2 \%$ for males and $10.6 \%$ for females $\left(\chi^{2}(1)=11.15 ; P=0.001\right)$ and peaked in the $65-74$ years age group to $25.1 \%$ for males and $17.4 \%$ for females $\left(\chi^{2}(1)=70.46 ; P<0.001\right)$. A schema that presents the inclusion of participants is shown in Supplementary Figure S1 online.

\section{Evaluation of concordance}

A total of 10,464 complete twin pairs (singletons excluded) with information about their tinnitus (yes/no; pairs without tinnitus excluded) and information on zygosity (unknown zygosity excluded) were analyzed in the concordance study (Supplementary Figure S1 online). Twin pairs were considered concordant for tinnitus if they both reported having tinnitus regardless of laterality. Pairs were considered to be bilaterally concordant only if both twins reported bilateral tinnitus. Pairs were considered discordant when one twin reported bilateral tinnitus and the other twin reported no tinnitus or unilateral tinnitus. Using the same reasoning, unilateral concordant pairs were identified. Casewise concordance is the risk of one twin being affected if their twin partner is affected. Concordance stratified for sex was calculated only for same-sex twin pairs. The methodology for zygosity determination and validity (DNA-based and intrapair similarity algorithm) within the Swedish Twin Registry was previously described. ${ }^{10}$ The accuracy of using answers to self-reported similarity questions for determining zygosity has repeatedly been found to be approximately $98 \%$ for the investigated cohorts. ${ }^{11}$ The proportion of DNA-based or intrapair similarity algorithm zygosity determination within SALT and STAGE samples used in the present concordance study is reported in Supplementary Table S1 online. Consistent with the more recent establishment of STAGE, more algorithmbased zygosity determinations are available for STAGE than SALT.

\section{Biometric modeling of tinnitus risk in twin pairs}

Quantitative models were analyzed to estimate the magnitude of genetic and environmental influences that explain the variance in tinnitus liability. ${ }^{12,13}$ The general approach analyzes disease covariance between members of $\mathrm{MZ}$ and $\mathrm{DZ}$ pairs to separate the variation into a sum of components: additive genetic effects (A), dominant genetic effects (which model deviations of the heterozygote genotype from the mean of the homozygote genotype) (D), common environmental effects (C), and individually unique environmental effects (E). The genetic parameters of the model were specified on the basis of biologic relation between the co-twins. Within-pair covariance of liability is expressed as $\kappa \operatorname{var}(\mathrm{A})+\gamma \operatorname{var}(\mathrm{D})+\operatorname{Var}(\mathrm{C})$, where $\kappa=\gamma=1$ for MZ pairs and $\kappa=1 / 2$ and $\gamma=1 / 4$ for DZ pairs. ${ }^{12,13}$ Owing to statistical issues of identifiability, A, D, and C could not be estimated simultaneously. ${ }^{13}$ Therefore, a series of models were tested to allow the sequential testing of the significance of specific parameters. Measurement error was estimated in $\mathrm{E}$ because this component of variance does not contribute to within-pair resemblance. Dominance effects are, typically, biologically implausible in the absence of additive effects. The primary models are the ACE and ADE models, as well as their submodels AE, CE, and E. We tested for equal thresholds (i.e., normal quantiles of prevalence) between $\mathrm{MZ}$ and $\mathrm{DZ}$ twins, which is equivalent to assuming that the risk of disease does not differ by zygosity. The biometric modeling approach we applied did not correct for possible bias due to censoring at follow-up. For instance, some twins may not have experienced tinnitus yet. We assessed the fit of the submodels during each decade by using the Akaike information criterion. The code can be made available upon request. 


\section{Statistical analyses}

Analyses were repeated at least twice by independent researchers. Differences between the tinnitus and nontinnitus group were tested using the chi-squared test. The significance level was set at $\alpha=0.05$. Statistical analyses were performed using SPSS 22 and R 3.1.3.

\section{RESULTS}

Higher concordance of tinnitus in monozygotic twins

A total of 10,464 twin pairs concordant or discordant for tinnitus were investigated. Table 1 shows the results of the concordance analysis for STAGE and SALT combined. The casewise concordance was greater for MZ twins than for DZ twins (MZ: 32\%, 95\% CI 28-36; DZ: 20\%, 95\% CI 16-24). Concordance was similar between DZ twins of the same sex (SS-DZ) than between OS-DZ twins (SS-DZ: 20\%, 95\% CI 16-24; OS-DZ: 19\%, 95\% CI 16-23). Unlike prevalence, overall concordance of tinnitus in male $\mathrm{MZ}$ twins was similar to that of female $\mathrm{MZ}$ twins (male MZ: 25\%, 95\% CI 20-30; female MZ: 23\%, 95\% CI 19-28), and so was the concordance between male SS-DZ and female SS-DZ twins (male SS-DZ: 11\%, 95\% CI 8-14; female SS-DZ: 13\%, 95\% CI 11-17) (Table 1).

\section{Higher concordance rate of bilateral, but not unilateral, tinnitus in $\mathrm{MZ}$ twins}

When dividing the subjects according to tinnitus laterality, no difference in concordance was found for unilateral tinnitus in MZ twins when compared to DZ twins (MZ: 25\%, 95\% CI 2030; DZ: 19\%, 95\% CI 15-24), and this was also the case when stratified by gender (male MZ: 23\%, 95\% CI 17-30; male DZ: 16\%, 95\% CI 11-22; female MZ: 19\%, 95\% CI 14-25; female DZ: 14\%, 95\% CI 10-19) (Table 1). However, bilateral tinnitus had a greater concordance rate in MZ twins in comparison with DZ twins (MZ: 49\%, 95\% CI 44-53; DZ: 30\%, 95\% CI 26-35). This effect was significant in both genders (male MZ: 40\%, 95\% CI 34-45; male DZ: 15\%, 95\% CI 11-20; female MZ: 38\%, 95\% CI 33-44; female DZ: 23\%, 95\% CI 19-29) (Table 1). Table 2 presents concordance scores for bilateral tinnitus cases for the whole group and for individuals younger than 40 years. Younger MZ females had greater concordance than DZ females (MZ: 39\%, 95\% CI 32-47; DZ: 20\%, 95\% CI 12-30), whereas this was not observed for males (MZ: 39\%, 95\% CI 31-48; DZ: $32 \%, 95 \%$ CI 22-43).

\section{Heritability of tinnitus is greater in bilateral cases}

We next evaluated the heritability $(\mathrm{H} 2)$ of overall tinnitus (Table 3). Men appeared to have heritability more similar to that of overall tinnitus than women (men: $\mathrm{H} 2=41 \%, 95 \%$ CI 35-47; women: $\mathrm{H} 2=44 \%$, 95\% CI 26-61), with the contribution of the shared environment being almost null. However, when tinnitus subtypes were assessed according to laterality, men displayed greater heritability values for bilateral tinnitus than unilateral tinnitus (bilateral: 68\%, 95\% CI 63-73; unilateral: 29\%, 95\% CI 8-50), with a lack of contribution from the shared environment, which was not the case for women (bilateral: $41 \%, 95 \%$ CI 23-58; unilateral: 25\%, 95\% CI 5-46). Interestingly, when individuals younger than 40 years of age were selected, the heritability of tinnitus in women increased to $62 \%(22-100)$,

Table 1 Concordance of unilateral and bilateral tinnitus according to gender

\begin{tabular}{|c|c|c|c|c|c|c|c|c|}
\hline & & $\begin{array}{c}\text { Both } \\
\text { genders } \mathrm{MZ}\end{array}$ & SS-DZ & OS-DZ & Male MZ & SS-DZ & Female MZ & SS-DZ \\
\hline & Discordant & 1,380 & 1,897 & 1,959 & 653 & 988 & 727 & 909 \\
\hline \multirow[t]{2}{*}{ Unilateral } & Concordant & 41 & 58 & 42 & 17 & 27 & 24 & 31 \\
\hline & Discordant & 789 & 917 & 912 & 360 & 428 & 429 & 489 \\
\hline & Discordant & 885 & 1,178 & 1,199 & 458 & 671 & 427 & 507 \\
\hline & $\mathrm{CC}(\mathrm{Cl})$ & $0.49(0.44,0.53)$ & $0.30(0.26,0.35)$ & $-(-,-)$ & $0.40(0.34,0.45)$ & $0.15(0.11,0.20)$ & $0.38(0.33,0.44)$ & $0.23(0.19,0.29)$ \\
\hline
\end{tabular}

$95 \% \mathrm{Cl}$ shown in parentheses.

CC, casewise concordance; MZ, monozygotic pairs; OS-DZ, dizygotic pairs of opposite sex; SS-DZ, dizygotic pairs of the same sex.

Table 2 Number of concordant and discordant pairs and casewise concordance for bilateral tinnitus by gender and zygosity before age at follow-up

\begin{tabular}{lccccccc} 
Age group & Zygosity & $\begin{array}{c}\text { Male } \\
\text { concordant }\end{array}$ & $\begin{array}{c}\text { Male } \\
\text { discordant }\end{array}$ & $\begin{array}{c}\text { Male CC } \\
\mathbf{( 9 5 \% ~ C l )}\end{array}$ & $\begin{array}{c}\text { Female } \\
\text { concordant }\end{array}$ & $\begin{array}{c}\text { Female } \\
\text { discordant }\end{array}$ & $\begin{array}{c}\text { Female CC } \\
(\mathbf{9 5} \% \mathbf{C l})\end{array}$ \\
\hline Before age 40 & $\mathrm{MZ}$ & 37 & 115 & $0.39(0.31,0.48)$ & 45 & 139 & $0.39(0.32,0.47)$ \\
& $\mathrm{DZ}$ & 18 & 79 & $0.32(0.22,0.43)$ & 13 & 108 & $0.20(0.12,0.30)$ \\
Before age 85 & $\mathrm{MZ}$ & 101 & 458 & $0.40(0.34,0.45)$ & 69 & 427 & $0.38(0.33,0.44)$ \\
& $\mathrm{DZ}$ & 60 & 671 & $0.15(0.11,0.20)$ & 44 & 507 & $0.23(0.19,0.29)$
\end{tabular}

Values are provided for groups younger than 40 years and compared with the whole cohort (before age 85 ). 95\% Cl shown in parentheses.

CC, casewise concordance; DZ, dizygotic pairs; MZ, monozygotic pairs. 
Table 3 Heritability of unilateral and bilateral tinnitus according to gender

\begin{tabular}{lccrr} 
& Gender & H2 & C2 & e2 \\
\hline All & Both & $0.43(0.39,0.47)$ & $0.0(-,-)$ & $0.0(-,-)$ \\
& Male & $0.41(0.35,0.47)$ & $0.01(0.0,0.15)$ & $0.56(0.50,0.62)$ \\
Unilateral & Female & $0.44(0.26,0.61)$ & $0.34(0.22,0.46)$ & $0.39(0.34,0.44)$ \\
& Both & $0.27(0.12,0.42)$ & $0.35(0.18,0.52)$ & $0.36(0.29,0.43)$ \\
& Male & $0.29(0.08,0.50)$ & $0.33(0.16,0.50)$ & $0.41(0.35,0.48)$ \\
Bilateral & Female & $0.25(0.05,0.46)$ & $0.13(0.02,0.24)$ & $0.31(0.27,0.34)$ \\
& Both & $0.56(0.43,0.69)$ & $0.0(-,-)$ & $0.33(0.27,0.37)$ \\
& Male & $0.68(0.63,0.73)$ & $0.29(0.14,0.44)$ & $0.30(0.25,0.35)$ \\
\hline
\end{tabular}

$95 \% \mathrm{Cl}$ shown in parentheses.

C2, shared; e2, unique environmental effects; $\mathrm{H} 2$, heritability.

Table 4 Heritability of bilateral tinnitus by gender and age at follow-up

\begin{tabular}{lcccccc} 
Age groups & Male H2 & C2 & e2 & Female H2 & C2 & e2 \\
\hline Before age 40 & $0.22(0.0,0.62)$ & $0.38(0.04,0.75)$ & $0.39(0.27,0.50)$ & $0.62(0.22,1.0)$ & $0.03(0.0,0.41)$ & $0.35(0.25,0.44)$ \\
Before age 85 & $0.68(0.63,0.73)$ & $0.0(-,-)$ & $0.33(0.27,0.37)$ & $0.41(0.23,0.58)$ & $0.29(0.14,0.44)$ & $0.30(0.25,0.35)$ \\
\hline
\end{tabular}

95\% Cl shown in parentheses.

C2, shared; e2, unique environmental effects are provided for groups younger than 40 years and compared to the whole cohort (before age 85 ); $\mathrm{H} 2$, heritability.

although this was quite variable (Table 4). Again, with greater heritability scores, the contribution of the shared environment (C2) appeared minimal in young women $(3 \%, 95 \%$ CI $0-41)$.

\section{DISCUSSION}

Twin concordance data are used to measure the relative importance of heredity and environment for the development of specific quantitative traits. In a narrow sense, heritability considers the additive portion of the genetic variability in relation to the phenotypic variability. ${ }^{14,15}$ For the first time, we demonstrated the extent to which age and gender may affect the genetic and environmental components in individuals with bilateral tinnitus, providing a relatively high heritability estimate for bilateral tinnitus of 0.68 for men and 0.62 for women younger than 40 years.

The prevalence data reported here (14.9\%) are in agreement with those from several previous reports that used large samples. ${ }^{16}$ These reports also demonstrated that the incidence of tinnitus increases with age, ${ }^{17-19}$ with a peak during the seventh decade of life for men and the eighth decade for women. Although there is no agreement on a potential gender bias, ${ }^{4}$ our data indicate that men are more prone to developing tinnitus, whether unilateral or bilateral. In contrast to these results, heritability scores of overall tinnitus appeared similar between men and women. Because tinnitus is age-dependent, an additive effect of environmental factors and aging on tinnitus development is conceivable. This effect could dilute the genetic contribution for the oldest individuals, making it very difficult to segregate tinnitus from other comorbid conditions, such as agerelated hearing loss.

The heritability scores we identified for overall tinnitus (0.43) are within the range found for other complex disorders related to the brain or central nervous system, including major depression (0.37), Parkinson disease (0.34), and Alzheimer disease (0.48)..$^{20}$ As individuals age, their exposures to the environment, emotional stress, and age-related diseases are potentially confounding variables. An advantage of our study was that it was able to discriminate between individuals with unilateral or bilateral tinnitus and it investigated heritability according to the laterality of the tinnitus percept and gender. Heritability values for bilateral tinnitus reached 0.68 in men, which is close to what has been found for other brain disorders, such as attention-deficit hyperactivity disorders, autism, and schizophrenia (0.76, 0.71 , and 0.81 respectively), for which a significant genetic contribution has been acknowledged. ${ }^{20}$ In contrast, results obtained for unilateral tinnitus indicated an equal distribution of genetic, common shared, and unique environmental factors. A genetic factor would thus be predisposing for bilateral tinnitus, whereas environmental factors such as noise exposure would be predisposing for unilateral tinnitus. Future studies will need to address the differences in tinnitus etiology between unilateral and bilateral cases and take gender into account. Overall, although tinnitus as a whole might be considered environmentally driven, the present study indicates that bilateral tinnitus might constitute a genetic subtype.

Several genotyping studies failed to reveal consistent associations of specific genes (e.g., GDNF, BDNF, KCTD12, KCNE1, SLC6A4) with tinnitus. ${ }^{4}$ Most of these studies were case-controlled, underpowered, and performed during the pregenomic era, with insufficiently characterized auditory performance. In cases where associations were found, these were not replicated in an independent cohort. Selection strategies are becoming a crucial approach in sequencing studies. For instance, selection of severe phenotypes in combination with specific subtypes with higher concordance has been successfully used to decipher rare variants of major depressive disorders. ${ }^{21}$ According to the 
present study, to investigate the genetic underpinnings of tinnitus the preferred phenotype would be severe bilateral tinnitus since this group has been shown to carry the highest heritability. Because chronic and persistent tinnitus in young persons is not a common condition, multicase families with early onset of severe or debilitating bilateral tinnitus should be prioritized for exome/genome sequencing studies. Another reason to concentrate future genomic work on younger individuals is that the copy-number variations accumulate with aging, ${ }^{22}$ and structural changes in DNA will increase the background noise in exome data sets. Therefore, selecting women younger than 40 years with bilateral tinnitus, a population in which heritability reaches 0.62 , might be fruitful.

Sex-specific characteristics may be related to differences in hormone exposure during fetal development. The influence of small fetal hormonal changes on physiology and behavior has been revealed in animal studies using rodents with large litters ${ }^{23}$ that have demonstrated the transfer of hormones to neighboring fetuses. ${ }^{24,25}$ The transfer ultimately leads to changes in adulthood, a phenomenon called "fetal programming" that reveals potential fetal influences on exogenous compounds such as endocrine-disrupting chemicals interfering in nonmonotonic dose responses. ${ }^{26}$ Endogenous endocrine homeostasis may lead to greater risk of developing disorders in adulthood. ${ }^{27,28}$ In humans, this prenatal hormone transfer (the transfer of hormones between fetuses in utero) can be studied in opposite-sex (OS) or same-sex (SS) twins, whereby the masculinization of specific traits during fetal life determines the adult characteristics (e.g., sensation seeking, breast cancer predisposition). ${ }^{29,30}$ Such effects have already been revealed in the context of the auditory system, whereby the presence of a male co-twin "masculinizes" the ear of females and reduces the number of otoacoustic emissions (OAEs). ${ }^{31,32}$ Our study indicates that OS-DZ twins display similar concordance of tinnitus (either unilateral or bilateral) compared with female or male SS twins, suggesting that alterations in fetal hormone levels would be unlikely to result in greater risks of developing tinnitus in adulthood. Thus, additional research is needed to investigate whether a particular trigger is responsible for the emergence of bilateral tinnitus in a genetically predisposed adult subject or whether the condition would occur in the absence of causative factors as a result of multiple genetic interactions.

A weakness in the present cross-sectional study is the lack of information regarding age at tinnitus onset. Significant bias in the heritability estimates might emerge from the fact that a fraction of the subjects at risk may not have experienced tinnitus at the time of follow-up. ${ }^{33,34}$ Future twin studies of tinnitus taking into account age of onset might exclude this censoring bias and optimize the estimates of heritability. Because tinnitus is heterogeneous, this study was also limited by the lack of in-depth characterization of tinnitus features, the accompanying emotional burden, and the auditory profiles. Regarding the latter, genetically based or acquired hearing loss is a major risk factor for developing tinnitus. ${ }^{17}$ Clinical observations have shown that $75 \%$ of subjects with confirmed mutations in the mitochondrial DNA also have high-frequency hearing loss accompanied by tinnitus. ${ }^{35}$ Human late-onset, progressive, sensorineural hearing loss and vestibular disorder DFNA9 harbor mutations in the $\mathrm{COCH}$ gene encoding the highly conserved protein COCHLIN expressed in the inner ear. ${ }^{36}$ All patients with DFNA9-induced hearing loss also experience tinnitus. Tinnitus is also a common symptom of several otological disorders, such as Ménière disease ${ }^{37}$ and otosclerosis. ${ }^{38}$ Therefore, the question of whether our concordance results are influenced by hearing loss or other auditory disorders is justified. However, it is very difficult to separate the genetic effect associated with hearing loss from that associated with tinnitus only.

As a consequence, a genetic basis for bilateral tinnitus may originate from an accompanying hearing loss, both determined by hereditary factors. In-depth phenotyping of auditory performance that extends beyond the classic pure-tone audiometry would be necessary to determine with precision the relationship between tinnitus and hearing loss in twins. For instance, a comparison of hearing performance in younger MZ twins discordant for tinnitus could clarify the dependence of bilateral tinnitus on hearing loss. This information could eventually be obtained through linkage with medical registries, but the lack of national clinical guidelines for the assessment of tinnitus in Sweden may significantly decrease the statistical power of such analyses. Moreover, most audiometric measurements have probably been performed between $125 \mathrm{~Hz}$ and $8 \mathrm{kHz}$, which are frequencies mainly relevant for human communication. However, because the hearing abilities of healthy individuals reach up to $20 \mathrm{kHz}$, high-frequency audiometry would be required when diagnosing subjects with tinnitus in order to obtain more precise information about the existence of auditory deficits. It has also been questioned whether pure-tone audiometry is sufficiently sensitive to detect auditory deficits because more advanced measures using auditory brainstem responses are capable of revealing potential damage in the inner ear evidenced by a decrease in cochlear output, ${ }^{39}$ which has been correlated with tinnitus in animal models..$^{40}$ As a consequence, the current methodologies used in the clinics for the assessment of auditory functions might not suffice for identifying a potential peripheral origin of tinnitus generation.

Overall, the present study confirms that tinnitus is multifactorial and includes both genetic and nongenetic factors. Our results strongly support the minor contribution of a common shared environment in the etiology of tinnitus per se. In addition, the obtained data indicate that genes play an important role in tinnitus, particularly in bilateral cases. We therefore propose that a genetic predisposition might be important for the development of some forms of tinnitus, and that nongenetic factors possibly have a predominant role in controlling whether a genetically predisposed subject will progress to tinnitus.

\section{SUPPLEMENTARY MATERIAL}

Supplementary material is linked to the online version of the paper at http://www.nature.com/gim 


\section{ACKNOWLEDGMENTS}

We are grateful to the Swedish Twin Registry for providing access to the STAGE and SALT data and to Ralf Kuja-Halkola for scientific advice. We thank Proof-Reading-Services.com for language editing services. C.R.C. has received funding from Vetenskapsrådet, Lars Hiertas Minne, Magnus Bergvalls Stiftelserna, Tysta Skolan, and Karolinska Institutet. B.C. has received funding from the Swedish Medical Council, Tysta Skolan, and Karolinska Institutet. The work was supported by an independent research program funded under the Biomedicine and Molecular Biosciences European Cooperation in Science and Technology (COST) Action framework (TINNET, BM1306).

\section{DISCLOSURE}

The authors declare no conflict of interest.

\section{REFERENCES}

1. Dobie RA. Depression and tinnitus. Otolaryngol Clin North Am 2003;36:383388.

2. Heller AJ. Classification and epidemiology of tinnitus. Otolaryngol Clin North Am 2003;36:239-248.

3. Cederroth $C R$, Canlon $B$, Langguth $B$. Hearing loss and tinnitus-are funders and industry listening? Nat Biotechno/ 2013;31:972-974.

4. Lopez-Escamez JA, Bibas T, Cima RF, et al. Genetics of tinnitus: an emerging area for molecular diagnosis and drug development. Front Neurosci 2016;10:377.

5. Baguley D, McFerran D, Hall D. Tinnitus. Lancet 2013;382:1600-1607.

6. Langguth B, Kreuzer PM, Kleinjung T, De Ridder D. Tinnitus: causes and clinical management. Lancet Neuro/ 2013;12:920-930.

7. Kvestad E, Czajkowski N, Engdahl B, Hoffman HJ, Tambs K. Low heritability of tinnitus: results from the second Nord-Trøndelag health study. Arch Otolaryngol Head Neck Surg 2010;136:178-182.

8. Hendrickx JJ, Huyghe JR, Demeester K, et al. Familial aggregation of tinnitus: a European multicentre study. B-ENT 2007;3(suppl 7):51-60.

9. Lichtenstein P, Sullivan PF, Cnattingius S, et al. The Swedish Twin Registry in the third millennium: an update. Twin Res Hum Genet 2006;9:875-882.

10. Magnusson PK, Almqvist C, Rahman I, et al. The Swedish Twin Registry: establishment of a biobank and other recent developments. Twin Res Hum Genet 2013;16:317-329.

11. Lichtenstein P, De Faire U, Floderus B, Svartengren M, Svedberg P, Pedersen NL. The Swedish Twin Registry: a unique resource for clinical, epidemiological and genetic studies. J Intern Med 2002;252:184-205.

12. Neale MC, Cardon LR. Methodology for Genetic Studies of Twins and Families. Kluwer Academic Publishers: Dordrecht, The Netherlands, 1992

13. Sham P. Statistics in Human Genetics. John Wiley \& Sons: Chichester, UK, 1998.

14. Bell AE. Heritability in retrospect. J Hered 1977;68:297-300.

15. Kang KW, Christian JC, Norton JA Jr. Heritability estimates from twin studies. I. Formulae of heritability estimates. Acta Genet Med Gemellol (Roma) 1978;27:39-44.

16. McCormack A, Edmondson-Jones M, Somerset S, Hall D. A systematic review of the reporting of tinnitus prevalence and severity. Hear Res 2016;337:70-79.

17. Gopinath B, McMahon CM, Rochtchina E, Karpa MJ, Mitchell P. Incidence, persistence, and progression of tinnitus symptoms in older adults: the Blue Mountains Hearing Study. Ear Hear 2010;31:407-412.

18. Shargorodsky J, Curhan GC, Farwell WR. Prevalence and characteristics of tinnitus among US adults. Am J Med 2010;123:711-718.

19. Park B, Choi HG, Lee HJ, et al. Analysis of the prevalence of and risk factors for tinnitus in a young population. Otol Neurotol 2014;35:1218-1222.

20. van Dongen J, Slagboom PE, Draisma HH, Martin NG, Boomsma DI. The continuing value of twin studies in the omics era. Nat Rev Genet 2012;13:640 653.

21. CONVERGE consortium. Sparse whole-genome sequencing identifies two loci for major depressive disorder. Nature 2015;523:588-591.
22. Forsberg LA, Rasi C, Razzaghian HR, et al. Age-related somatic structural changes in the nuclear genome of human blood cells. Am J Hum Genet 2012;90:217-228.

23. Ryan BC, Vandenbergh JG. Intrauterine position effects. Neurosci Biobehav Rev 2002;26:665-678.

24. vom Saal FS, Grant WM, McMullen CW, Laves KS. High fetal estrogen concentrations: correlation with increased adult sexual activity and decreased aggression in male mice. Science 1983;220:1306-1309.

25. Vom Saal FS, Dhar MG. Blood flow in the uterine loop artery and loop vein is bidirectional in the mouse: implications for transport of steroids between fetuses. Physiol Behav 1992;52:163-171.

26. Vandenberg LN, Colborn T, Hayes TB, et al. Hormones and endocrine-disrupting chemicals: low-dose effects and nonmonotonic dose responses. Endocr Rev 2012;33:378-455

27. Cederroth $C R$, Nef $S$. Fetal programming of adult glucose homeostasis in mice. PLoS One 2009;4:e7281.

28. vom Saal FS, Timms BG, Montano MM, et al. Prostate enlargement in mice due to fetal exposure to low doses of estradiol or diethylstilbestrol and opposite effects at high doses. Proc Natl Acad Sci USA 1997;94:2056-2061.

29. Resnick SM, Gottesman II, McGue M. Sensation seeking in opposite-sex twins: an effect of prenatal hormones? Behav Genet 1993;23:323-329.

30. Hsieh CC, Lan SJ, Ekbom A, Petridou E, Adami HO, Trichopoulos D. Twin membership and breast cancer risk. Am J Epidemiol 1992;136:1321-1326.

31. McFadden D. A masculinizing effect on the auditory systems of human females having male co-twins. Proc Natl Acad Sci USA 1993;90:11900-11904.

32. McFadden D, Loehlin JC, Pasanen EG. Additional findings on heritability and prenatal masculinization of cochlear mechanisms: click-evoked otoacoustic emissions. Hear Res 1996;97:102-119.

33. Scheike TH, Hjelmborg JB, Holst KK. Estimating twin pair concordance for age of onset. Behav Genet 2015;45:573-580.

34. Holst KK, Scheike TH, Hjelmborg JB. The liability threshold model for censored twin data. Computational statistics and Data Analysis. 2016;93:324-335.

35. Yano T, Nishio SY, Usami S; Deafness Gene Study Consortium. Frequency of mitochondrial mutations in non-syndromic hearing loss as well as possibly responsible variants found by whole mitochondrial genome screening. J Hum Genet 2014;59:100-106.

36. Verstreken M, Declau F, Wuyts FL, et al. Hereditary otovestibular dysfunction and Ménière's disease in a large Belgian family is caused by a missense mutation in the COCH gene. Otol Neurotol 2001;22:874-881.

37. Lopez-Escamez JA, Dlugaiczyk J, Jacobs J, et al. Accompanying symptoms overlap during attacks in Menière's disease and vestibular migraine. Front Neurol 2014;5:265.

38. Schaette R. Tinnitus in men, mice (as well as other rodents), and machines. Hear Res 2014:311:63-71.

39. Schaette R, McAlpine D. Tinnitus with a normal audiogram: physiological evidence for hidden hearing loss and computational model. J Neurosci 2011;31:13452-13457.

40. Rüttiger $L$, Singer W, Panford-Walsh $R$, et al. The reduced cochlear output and the failure to adapt the central auditory response causes tinnitus in noise exposed rats. PLoS One 2013;8:e57247.

This work is licensed under a Creative Commons Attribution-NonCommercial-ShareAlike 4.0 International License. The images or other third party material in this article are included in the article's Creative Commons license, unless indicated otherwise in the credit line; if the material is not included under the Creative Commons license, users will need to obtain permission from the license holder to reproduce the material. To view a copy of this license, visit http://creativecommons.org/licenses/ by-nc-sa/4.0/

(C) The Author(s) (2016) 